\title{
Recoupling mechanism for exotic mesons and baryons
}

\author{
Yu.A. Simonov \\ NRC "Kurchatov Institute" - ITEP, \\ B. Cheremushkinskaya 25, Moscow, 117259, Russia \\ E-mail: simonov@itep.ru
}

ABSTRACT: The infinite chain of transitions of one pair of mesons (channel I) into another pair of mesons (channel II) can produce bound states and resonances in both channels even if no interactions inside channels exist. These resonances which can occur also in meson-baryon channels are called channel-coupling (CC) resonances. A new mechanism of $\mathrm{CC}$ resonances is proposed where transitions occur due to a rearrangement of confining strings inside each channel - the recoupling mechanism. The amplitude of this recoupling mechanism is expressed via overlap integrals of the wave functions of participating mesons (baryons). The explicit calculation with the known wave functions yields the peak at $E=4.12 \mathrm{GeV}$ for the transitions $J / \psi+\phi \leftrightarrow D_{s}^{*}+\bar{D}_{s}^{*}$, which can be associated with $\chi_{c 1}(4140)$, and a narrow peak at $3.98 \mathrm{GeV}$ with the width $10 \mathrm{MeV}$ for the transitions $D_{s}^{-}+$ $D_{0}^{*} \leftrightarrow J / \psi+K^{*-}$, which can be associated with th recently discovered $Z_{c s}(3985)$.

KeYWords: Phenomenological Models

ARXIV EPRINT: 2011.12326 


\section{Contents}

1 Introduction $\quad 1$

2 The simplest case: only separable CC interaction 4

3 Equations for two channel amplitudes in the recoupling formalism 5

4 Recoupling mechanism for the meson-meson amplitudes $\quad 8$

5 Recoupling mechanism for meson-baryon systems $\quad 9$

6 Physical amplitudes and their singularities in the recoupling process 11

$\begin{array}{lll}7 & \text { Numerical results and discussion } & 13\end{array}$

8 Conclusions and an outlook $\quad 15$

A The center-of-mass coordinates and average quark and antiquark energies $\begin{array}{ll}\text { in a hadron } & 16\end{array}$

$\begin{array}{ll}\text { B The channel-coupling coefficient } \bar{y}_{1234} & 16\end{array}$

$\begin{array}{ll}\text { C Oscillator parameters of hadron wave function } & 18\end{array}$

D Calculation of the cross sections $\left|f_{i k}\right|^{2}$ and pole positions 18

\section{Introduction}

The modern situation with the spectra of quarkonia and baryons requires the dynamical explanation of numerous extra states, which are not present in the one-channel spectra of a given meson [1]. ${ }^{1}$ A similar situation occurs in the excited baryon spectra. ${ }^{2}$ To be more precise, in the case of heavy quarkonia, i.e. states, which contain $c \bar{c}$ and $b \bar{b}$ pairs, the experimental data contain a number of charged $Z_{c}, Z_{b}$ and neutral $Y_{c}, Y_{b}$ states, which cannot be explained by the dynamics of $c \bar{c}$, or $b \bar{b}$ pairs alone, see [2] for review.

There are theoretical suggestions of different mechanisms [3-24], which should be taken into account. E.g. poles (resonances) in the meson-meson channels can occur due to strong interaction in these systems, and appear as additional poles in the $S$ matrix $[4,7,8,18,19]$

— the molecular-type approach.

\footnotetext{
${ }^{1}$ See also the update on 6th December 2019.

${ }^{2}$ M. Karliner and T. Skwarnicki, Pentaquarks, in [1].
} 
A similar in the choice of the driving channels $(Q \bar{q} \bar{Q} q)$, but different in the dynamics, is the approach of the tetraquark model $[5,10,12-14,17,20,24]$, see [25-27] for reviews. A more general approach contains features of both molecular and tetraquark models - the hybridized tetraquark model [28]. One of the basic features of these models (as well as of the hadron-hadron interaction in general) is the determination of the dynamics in the system of two white objects. In the case of a deep tetraquark state one can disregard the twobody white asymptotics with some accuracy,however in the molecular case and in general one needs a classification of possible dynamical exchanges $V_{h h}$ between two white hadrons, taking into account the full gauge invariance and the confinement via general Wilson loop representation, which is now standard. It is clear that one-gluon exchange and its spin versions (spin-spin,spin-orbit,tensor) are not present in $V_{h h}$, while two-gluon exchange is the glueball exchange. The "exchange of confining interaction" is not a local potential but the transition of two white objects into a common white one with $q \bar{q}$ annihilations before and after, e.g. $\pi+\pi \rightarrow \rho \rightarrow \pi+\pi$. In a similar way one obtains (actually nonlocal) $t$-channel and $u$-channel hadron exchanges. A special role is played by the Coulomb (not color Coulomb) interaction, which always exists locally in addition to listed above interactions. It is important that all types of interaction between white objects (except for Coulomb) are short ranged for massive exchanged hadrons and do not produce narrow singularities by themselves. Our purpose in this paper is to find the dynamical origin of relatively narrow peaks nearby threshold which occur due to transformation of one white pair into another arbitrary number of times and to find what is the transformation itself. As we shall see, we shall meet a completely different type of transformation — we shall call it the recoupling and explain this mechanism in some detail. As a result we shall have here a new theoretical treatment of hadron-hadron interaction, suggesting a simple and quite general mechanism for exotic peaks in mesons and baryons - the recoupling mechanism.

In contrast to the approaches, where white-white interaction in the one-channel system, (e.g. in meson-meson) is generating resonances, we propose the dynamical picture, where the summed up transitions from one channel to another (without interaction inside channels) can be strong enough to produce resonances nearby thresholds. The specific feature of this interaction is that it depends strongly on the wave functions of both channels, entering in the overlap integral of the transition matrix element, which measures the amplitude of the transition between the initial $q \bar{Q}+\bar{q} Q$ and final $q \bar{q}+Q \bar{Q}$ states.

In what follows we are exploiting the channel-coupling $(\mathrm{CC})$ interaction in the form of the energy-dependent recoupling Green's functions as a possible origin of extra states the recoupling mechanism.

Indeed, more than 30 years ago, the present author participated in the systematic study of $\mathrm{CC}$ effects in the spectra of hadrons, nuclei and atoms [29]. It was found there, that the CC interaction defined by the Transition Matrix Element (TME) is able to produce resonances (poles) of its own, if TME is strong enough, i.e. if the corresponding TME satisfies certain conditions, similar to that for one-channel potential.

We show below, that at the basis of this recoupling process lies a simple picture of the string recoupling between the same systems of quarks and antiquarks, which does not need neither energy nor additional interaction, and is simply a kind of topological transformation of two confining strings with fixed ends into another pair of strings - the string recoupling. 




Figure 1. The transition of the mesons $\left(q_{1} \bar{q}_{2}\right)+\left(q_{3} \bar{q}_{4}\right) \leftrightarrow\left(q_{1} \bar{q}_{4}\right)+\left(\bar{q}_{2} q_{3}\right)$ via recoupling of the confining strings.

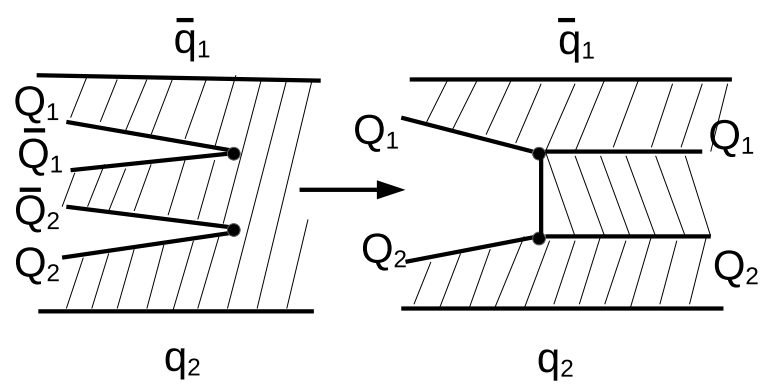

Figure 2. The mechanism of string recoupling via double string-breaking process at the points shown by thick dots.

One can see in figure 1 the confining regions (the crossed areas) for the bound states of quark-antiquark (mesons) $q_{1} \bar{q}_{2}$ and $q_{3} \bar{q}_{4}$ in the l.h.s. of the figure 1, which is transformed in the middle part of figure 1 into the confining region between $q_{1} \bar{q}_{4}$ on the plane of the figure, and "the confining bridge" - the double-crossed area between $\bar{q}_{2} q_{3}$. The r.h.s. of the figure 1 is the same as the l.h.s. As the result the transition is $\left(q_{1} \bar{q}_{2}\right)+\left(q_{3} \bar{q}_{4}\right) \leftrightarrow\left(q_{1} \bar{q}_{4}\right)+\left(\bar{q}_{2} q_{3}\right)$. It is interesting to understand what kind of vertices are responsible for this transition, and to this end we demonstrate in figure 2 below the possible construction of the "confining bridge" in the figure 1 by cutting the confining film and turning up the middle piece.

We show in this way that topologically this process is equivalent to the double string breaking, and numerically is defined by the everlap integral of participating hadron wave functions. This mechanism is quite general and can work for meson-meson, meson-baryon, baryon-baryon states. In particular it can work for some of $X, Y$ and $Z$ states of heavy quarkonia, like $Z_{c}(3900)$ and $Z_{c}(4020)$.

It is a purpose of the present paper to exploit this formalism for the case of extra states in meson-meson or meson-baryon spectra and define possible resonances and thresholds, and further on to apply this formalism to the case of pentaquark states like $P_{c}(4312), P_{c}(4440), P_{c}(4457)$.

Our main procedure will be the calculation of TME using realistic wave functions of $c \bar{c}, b \bar{b}, c \bar{u}$ systems, as well as approximate for baryon systems. Using those we calculate the 
resulting Green's functions and resonance positions and compare them with experiment. The plan of the paper is as follows.

In the next section we introduce the reader to the method by solving a simplified twochannel problem with a separable potential. Section 3 is devoted to the explicit formulation of the recoupling mechanism, section 4 contains application to the meson-meson channel, and section 5 considers the meson-baryon case. Section 6,7 are devoted to the analysis of the physical structure and numerical results and discussion, while section 7 contains conclusions and an outlook.

\section{The simplest case: only separable CC interaction}

Suppose we have two channels 1 and 2 with thresholds $E_{1}$ and $E_{2}$ and the CC interaction is separable

$$
V_{12}\left(p_{1}, p_{2}\right)=-\lambda v_{1}\left(p_{1}\right) v_{2}\left(p_{2}\right)=V_{21} .
$$

The Schroedinger-like (possibly relativistic) equations are

$$
\left(T_{1}-E\right) \varphi_{1}+V_{12} \varphi_{2}=0, \quad\left(T_{2}-E\right) \varphi_{2}+V_{21} \varphi_{1}=0
$$

and can be reduced to the equation

$$
\left(T_{1}-E\right) \varphi_{1}+V_{121}(E) \varphi_{1}=0,
$$

where $V_{121}$ is

$$
V_{121}\left(p_{1}, p_{1}^{\prime} E\right)=-\lambda^{2} v_{1}\left(p_{1}\right) v_{1}\left(p_{1}^{\prime}\right) \int \frac{v_{2}^{2}(k) d^{3} k /(2 \pi)^{3}}{T_{2}(k)+E_{2}-E} .
$$

Solving (2.3) one obtains the equation for the eigenvalue $E$

$$
1=\lambda^{2} \int \frac{v_{1}^{2}(p) d^{3} p /(2 \pi)^{3}}{T_{1}(p)+E_{1}-E} \int \frac{v_{2}^{2}(k) d^{3} k /(2 \pi)^{3}}{T_{2}(k)+E_{2}-E},
$$

or

$$
1=\lambda^{2} I_{1}(E) I_{2}(E) .
$$

For $E_{1}<E_{2}$ one can put $E=E_{1}$ and get a condition for the existence of a bound state in our two-channel system [29].

$$
\lambda^{2} I_{1}(E) I_{2}(E) \geq 1 .
$$

One of the intriguing points now is how the bound state poles, or more generally, any poles appear when the interaction strength $\lambda$ is large enough. To this end we make a simplifying assumption about the form of $v_{i}(k)$ and write

$$
\text { a) } v_{i}^{2}(k)=\frac{1}{k^{2}+\nu_{i}^{2}} \quad \text { b) } v_{i}^{2}(k)=\exp \left(-\frac{k^{2}}{4 \beta_{i}^{2}}\right)
$$

where $\nu_{1}, \nu_{2}$ and $\beta_{1}, \beta_{2}$ are some constants. Assuming also the nonrelativistic kinematics $T_{i}=\frac{k_{1}^{2}}{2 \mu_{i}}$ one obtains in the case a)

$$
I_{i}(E)=\frac{\mu_{i}}{2 \pi\left(\nu_{i}-i \sqrt{2 \mu_{i} \Delta_{i}}\right)}, \quad \Delta_{i}=E-E_{i},
$$


and we have taken square root (2.9) on the physical Riemann sheet, $E=E+i \delta$. Hence the equation (2.5) for the poles (energy eigenvalues) is

$$
\left(\nu_{1}-i \sqrt{2 \mu_{1}\left(E-E_{1}\right)}\right)\left(\nu_{2}-i \sqrt{2 \mu_{2}\left(E-E_{2}\right)}\right)=C=\frac{\mu_{1} \mu_{2} \lambda^{2}}{4 \pi^{2}} .
$$

We shall be mostly interested in the poles around the threshold $E_{2}$ and therefore in the first approximation we replace the first factor on the l.h.s. of (2.10) by a constant, assuming, that $E_{2}-E_{1}$ has a large positive value, hence one can write for $k_{2}=\sqrt{\mu_{2}\left(E-E_{2}\right)}$ using $(2.10)$

$$
k_{2} \cong-i \nu_{2}+i \lambda^{\prime 2}, \quad \lambda^{\prime 2}=\frac{\mu_{1} \mu_{2} \lambda^{2}}{4 \pi^{2}\left(\nu_{1}-i \sqrt{2 \mu_{1}\left(E_{2}-E_{1}\right)}\right)} .
$$

From (2.1) one can see that the pole is originally (at $\lambda^{\prime}=0$ ) on the second $E_{2}$ sheet, $k_{2}=-i \nu_{2}$ and remains on the second $E_{2}$ sheet with increasing $\lambda^{\prime}$. Note, however, that since originally we have been on the $E_{1}$ first sheet, then $\operatorname{Im} \lambda^{\prime 2}>0$, and therefore $\operatorname{Re} k_{2}<0$, implying that the pole can be of the Breit-Winger type for $\operatorname{Re} \lambda^{\prime 2}>\nu_{2}$.

As will be shown below in section 4 , resonance production cross sections are proportional to the function

$$
\frac{d \sigma^{*}(E)}{d E}=\left|\frac{1}{1-\lambda^{2} I_{1}\left(E-E_{1}\right) I_{2}\left(E-E_{2}\right)}\right|^{2} k_{2}(E) .
$$

We can generalize this separable form to the relativistic case, when two hadrons with masses $m_{3}, m_{4}$, so that the denominators in (2.5) look as follows:

$$
\begin{aligned}
& T_{1}(p)+E_{1}-E \rightarrow \sqrt{p^{2}+m_{1}^{2}}+\sqrt{p^{2}+m_{2}^{2}}-E, \\
& T_{2}(p)+E_{2}-E \rightarrow \sqrt{k^{2}+m_{3}^{2}}+\sqrt{k^{2}+m_{4}^{2}}-E
\end{aligned}
$$

Here we have two thresholds $m_{1}+m_{2}$ and $m_{3}+m_{4}$, and we shall assume that $m_{1}+m_{2}<$ $m_{3}+m_{4}$.

Making the replacement $(2.13)$ in $I_{1}(E), I_{2}(E)$ one can calculate these functions and find the behaviour of the approximate cross section in (2.12).

\section{Equations for two channel amplitudes in the recoupling formalism}

In this section we discuss the Green's function of the system of two white (noninteracting) hadrons $h_{1}, h_{2}$, which can transform into another system of white hadrons $H_{3}, H_{4}$ and this transformation can occur infinite number of times $h_{1} h_{2} \rightarrow H_{3} H_{4} \rightarrow h_{1} h_{2} \rightarrow H_{3} H_{4} \rightarrow \ldots$

Denoting the transition amplitude $V\left(h_{1} h_{2} \rightarrow H_{3} H_{4}\right)=V^{+}\left(H_{3} H_{4} \rightarrow h_{1} h_{2}\right)$, and the corresponding Green's functions as $G_{h}, G_{H}$, we obtain the total Green's function $G_{\alpha \beta}$, e.g. $G_{h h}$

$$
\begin{aligned}
G_{h h} & =G_{h}+G_{h} V_{h H} G_{H} V_{H h} G_{h}+G_{h} V_{h H} G_{H} V_{H h} G_{h} V_{h H} G_{H} V_{H h} G_{h}+\ldots= \\
& =G_{h} \frac{1}{1-V_{h H} G_{H} V_{H h} G_{h}} ;
\end{aligned}
$$


as a result one obtains the equation, which defines all possible singularities of the physical amplitudes, including the resonance poles.

$$
1=V_{h H} G_{H} V_{H h} G_{h}
$$

Note, that the described above method of the channel coupling was proposed before in the nonrelativistic form by the Cornell group [30-33], and exploited for the analytic calculation of the charmonium spectra, where the $h_{1} h_{2}$ are strongly interacting quarks $c \bar{c}$. The subsequent development of this method in [34-36] has allowed to understand the nature of the $X(3872)$ [35] and $Z_{b}$ states [36]. For the light quarks this method requires the explicit knowledge of $q \bar{q}$ spectrum and wave functions, which are available in the QCD string approach [37-46].

Recently the same approach, called the relativistic Cornell-type formalism successfully explained the spectrum of light scalars $[47,48]$. In our present case we disregard the interaction of hadrons $h_{1}$ with $h_{2}$ and $H_{3}$ with $H_{4}$.

Both Green's function $G_{H}, G_{h}$ describe propagation of two noninteracting subsystems, but each of these hadrons can have its own nontrivial spectrum.

In the simplest case, e.g. $h=\pi \bar{\pi}, H=K \bar{K}$, the Green's functions of noninteracting particles are well known, see e.g. $[47,48]$ for the scalar $\pi \pi, K \bar{K}$ Green's functions with the fixed spatial distance between $\pi \pi$ or $K \bar{K}$, needed to define the transition matrix element.

Since each of $h_{i}$ or $H_{j}$ is a composite system consisting of $q \bar{q}$ or $q q q$ one must write the corresponding relativistic composite Green's function, using the path integral formalism, see [44-46] for a recent review.

As it is seen from (2.13), one needs the explicit form of the relativistic Green's function, consisting of two quark-antiquark mesons $h_{1}\left(q_{1}, \bar{Q}_{1}\right)$ and $h_{2}\left(\bar{q}_{2}, Q_{2}\right)$ with the zero total momentum $\mathbf{P}=0$, so that the c.m. momentum of $q_{1} \bar{Q}_{1}$ is $\mathbf{p}_{1}$, while for $\bar{q}_{2} Q_{2}$ it is $-\mathbf{p}_{1}$. As a result the wave function of the $h_{1} h_{2}$ system with $\mathbf{P}=0$ and c.m. coordinates $\mathbf{R}$ can be written as

$$
\Psi_{h_{1} h_{2}}(\mathbf{u}-\mathbf{x} ; \mathbf{y}-\mathbf{v})=e^{i \mathbf{p}_{1} \mathbf{R}(\mathbf{u}, \mathbf{x})-i \mathbf{p}_{1} \mathbf{R}(\mathbf{y}, \mathbf{v})} \psi_{1}(\mathbf{u}-\mathbf{x}) \psi_{2}(\mathbf{y}-\mathbf{v}) .
$$

At the same time the relativistic wave function of the hadrons $H_{3}, H_{4}, h_{H}\left(q_{1} \bar{q}_{2}\right), H_{4}\left(\bar{Q}_{1} Q_{2}\right)$ has the form

$$
\Psi_{h_{3} h_{4}}(\mathbf{u}-\mathbf{v} ; \mathbf{v}-\mathbf{y})=e^{i \mathbf{p}_{2} \mathbf{R}(\mathbf{u}, \mathbf{v})-i \mathbf{p}_{2} \mathbf{R}(\mathbf{x}, \mathbf{y})} \psi_{3}(\mathbf{u}-\mathbf{v}) \psi_{4}(\mathbf{x}-\mathbf{y}) .
$$

Here we have introduced the c.m. coordinates $\mathbf{R}$ of the hadrons, expressed via the average energies $\omega_{i}, \Omega_{i}$ of the quarks and antiquarks in the hadron [49]

$$
\begin{array}{ll}
\mathbf{R}(\mathbf{u}, \mathbf{x})=\frac{\omega_{1} \mathbf{u}+\bar{\Omega}_{1} \mathbf{x}}{\omega_{1}+\bar{\Omega}_{1}}, & \mathbf{R}(\mathbf{y}, \mathbf{v})=\frac{\bar{\omega}_{2} \mathbf{v}+\Omega_{2} \mathbf{y}}{\bar{\omega}_{2}+\Omega_{2}}, \\
\mathbf{R}(\mathbf{u}, \mathbf{v})=\frac{\omega_{3} \mathbf{u}+\bar{\omega}_{4} \mathbf{v}}{\omega_{3}+\bar{\omega}_{4}}, & \mathbf{R}(\mathbf{x}, \mathbf{y})=\frac{\bar{\Omega}_{3} \mathbf{x}+\Omega_{4} \mathbf{y}}{\bar{\Omega}_{3}+\Omega_{4}} .
\end{array}
$$

Here $\omega_{i}, \Omega_{i}$ are given in the appendix $\mathrm{A}$ of this paper. 
Next we must calculate the overlap matrix element of $\Psi_{h_{1} h_{2}}$ and $\Psi_{h_{3} h_{4}}$

$$
V_{12 \mid 34}\left(\mathbf{p}_{1}, \mathbf{p}_{2}\right)=\int \bar{y}_{1234} d^{3}(u-x) d^{3}(y-v) d^{3}(u-v) \Psi_{h_{1} h_{2}} \Psi_{h_{3} h_{4}}^{+} \cdot
$$

Introducing the Fourier component of the wave functions e.g. $\psi_{1}(\mathbf{u}-\mathbf{x})=$ $\int \tilde{\psi}_{1}\left(\mathbf{q}_{1}\right) e^{i \mathbf{q}_{1}(\mathbf{u}-\mathbf{x})} \frac{d^{3} q_{1}}{(2 \pi)^{3}}$, one obtains in the simple case when $q_{2}=q_{1}, Q_{2}=Q_{1}$

$$
\begin{aligned}
V_{12 \mid 34}\left(\mathbf{p}_{1}, \mathbf{p}_{2}\right)= & \int \frac{d^{3} \mathbf{q}_{1}}{(2 \pi)^{3}} \bar{y}_{1234} \tilde{\psi}_{1}\left(\mathbf{q}_{1}\right) \tilde{\psi}_{2}\left(\mathbf{q}_{1}+\mathbf{p}_{2}\right) \tilde{\psi}_{3}\left(-\mathbf{q}_{1}-\frac{\mathbf{p}_{2}}{2}-\mathbf{p}_{1} \frac{\omega_{1}}{\omega_{1}+\Omega_{1}}\right) \\
& \times \tilde{\psi}_{4}\left(\mathbf{q}_{1}-\frac{\mathbf{p}_{2}}{2}-\mathbf{p}_{1} \frac{\Omega_{1}}{\omega_{1}+\Omega_{1}}\right) .
\end{aligned}
$$

In (3.7), (3.8) we introduced the numerical recoupling coefficient $\bar{y}_{1234}$, which is discussed in appendix B.

The transition element (3.7) with the factor $\bar{y}_{1234}$, responsible for the recoupling of hadrons, shown in figure 1, has a simple structure. Indeed, as one can see in figure 1 , the creation of two string configurations in the intermediate confining strings position and back into the original configuration. One may wonder what is the explicit mechanism of this recoupling, and what are the vertices denoted by thick points in the figure 2 . To this end we note, that we have two strings on the r.h.s. of figure 2: string from $Q_{1}$ to $\bar{Q}_{2}$ and another from $\bar{q}_{1}$ to $q_{2}$; this position results from the double string decay (the l.h.s. of figure 2 ) with the subsequent rotation of the string between $Q_{1}$ and $\bar{Q}_{2}$ to the right, where this string is at some distance above the string between $\bar{q}_{1}, q_{2}$. One can associate the quantity $M(x, y)$ with this process and we must add this factor to $V_{12 \mid 34}$. Writing $M(x, y)=\sigma|\mathbf{x}-\mathbf{y}|$ in analogy with the one-point string decay described by the effective Lagrangian [50] for the string decay,

$$
\mathcal{L}_{s d}=\int d^{4} x \bar{\psi}(x) M(x) \psi(x)
$$

and replacing it with the numerical value $M_{\omega}$, similarly to [34-36], (see appendix B for details) one can write $y_{1234}=M_{\omega} \chi_{1234}$ in (3.8), with $\chi_{1234}$ describing the spin-isospin recoupling. Finally one obtains the expression for the whole combination

$$
\begin{aligned}
& N(E)=G_{h_{1} h_{2}} V_{12 / 34} G_{h_{3} h_{4}} V_{34 / 12} \\
& N(E)=\int \frac{d^{3} \mathbf{p}_{1}}{(2 \pi)^{3}} \frac{d^{3} \mathbf{p}_{2}}{(2 \pi)^{3}} \frac{V_{12 \mid 34}\left(\mathbf{p}_{1}, \mathbf{p}_{2}\right) V_{34 \mid 12}\left(\mathbf{p}_{1}, \mathbf{p}_{2}\right)}{\left(E_{1}\left(\mathbf{p}_{1}\right)+E_{2}\left(\mathbf{p}_{1}\right)-E\right)\left(E_{3}\left(\mathbf{p}_{2}\right)+E_{4}\left(\mathbf{p}_{2}\right)-E\right)} .
\end{aligned}
$$

The resulting singularities (square root threshold singularities and possible poles from the equation $N(E)=1$ ) can be found in the integral (3.11).

One can see, that the structure of the expression (3.11) is the same as in eq. (2.5), provided $V_{12 \mid 34}$ factorizes in factors $v_{1}\left(p_{1}\right) v_{2}\left(p_{2}\right)$, and consequently one expects the same behaviour of the cross sections as in (2.12).

At this point it is useful to introduce the approximate form of the wave functions in (3.8), which is discussed in [36]. Here we only give the simplest form of the Gaussian wave functions for the ground states of light, heavy-light and heavy quarkonia. One can write

$$
\tilde{\psi}_{i}(q)=c_{i} \exp \left(-\frac{q^{2}}{2 \beta_{i}^{2}}\right), \quad c_{i}^{2}=\frac{8 \pi^{3 / 2}}{\beta_{i}^{3}} ; \quad \int \tilde{\psi}_{i}^{2}(q) \frac{d^{3} q}{(2 \pi)^{3}}=1
$$


where $\beta_{i}$ was found in [34-36], see appendix $\mathrm{C}$, e.g. for ground states of bottomonium $\beta=1.27 \mathrm{GeV}$, for charmonium $\beta=0.7 \mathrm{GeV}$ and for $D, B$ mesons $\beta=0.48,0.49 \mathrm{GeV}$.

Inserting $\tilde{\psi}_{i}(q)$ in (3.12) into (3.8) and integrating over $d^{3} \mathbf{q}_{1}$ one obtains

$$
V_{12 \mid 34}\left(\mathbf{p}_{1} \mathbf{p}_{2}\right)=\bar{y}_{1234}\left(\prod_{i=1}^{4} c_{i}\right) \frac{\exp \left(-A P_{2}^{2}-B p_{1}^{2}-C \mathbf{p}_{1} \mathbf{p}_{2}\right)}{(2 \pi)^{3} 8 \pi^{3 / 2} a^{3 / 2}},
$$

where $a, A, B, C$ are

$$
\begin{aligned}
a= & \frac{1}{2} \sum_{i=1}^{4} \frac{1}{\beta_{i}^{2}} ; \quad A=\frac{1}{2}\left(\frac{1}{\beta_{2}^{2}}+\frac{1}{4 \beta_{3}^{2}}+\frac{1}{4 \beta_{4}^{2}}\right)-\frac{1}{4 a}\left(\frac{1}{\beta_{2}^{2}}+\frac{1}{2 \beta_{3}^{2}}+\frac{1}{2 \beta_{4}^{2}}\right)^{2} \\
B= & \frac{1}{2 \beta_{3}^{2}}\left(\frac{\omega_{1}}{\omega_{1}+\Omega_{1}}\right)^{2}+\frac{1}{2 \beta_{4}^{2}}\left(\frac{\Omega_{1}}{\omega_{1}+\Omega_{1}}\right)^{2}-\frac{1}{4 a}\left(\frac{1}{\beta_{3}^{2}} \frac{\omega_{1}}{\omega_{1}+\Omega_{1}}-\frac{1}{\beta_{4}^{2}} \frac{\Omega_{1}}{\omega_{1}+\Omega_{1}}\right)^{2} \\
C= & \frac{1}{2}\left(\frac{1}{\beta_{3}^{2}} \frac{\omega_{1}}{\left(\omega_{1}+\Omega_{1}\right)}-\frac{1}{\beta_{4}^{2}} \frac{\Omega_{1}}{\left(\omega_{1}+\Omega_{1}\right)}\right)-\frac{1}{2 a}\left(\frac{1}{\beta_{2}^{2}}+\frac{1}{2 \beta_{3}^{2}}+\frac{1}{2 \beta_{4}^{2}}\right) \\
& \times\left(\frac{\omega_{1}}{\beta_{3}^{2}\left(\omega_{1}+\Omega_{1}\right.}-\frac{\Omega_{1}}{\beta_{4}^{2}\left(\omega_{1}+\Omega_{1}\right.}\right) .
\end{aligned}
$$

The resulting $N(E)$ has the form

$$
N(E)=\frac{M_{\omega}^{2} \bar{\chi}_{1234}^{2}}{a^{3}\left(\prod_{i} \beta_{i}\right)^{3}(\pi)^{3}} \int \frac{d^{3} p_{1} d^{3} p_{2} \exp \left(-2 A p_{2}^{2}-2 B p_{1}^{2}-2 C \mathbf{p}_{1} \mathbf{p}_{2}\right)}{\left(E_{1}\left(p_{1}\right)+E_{2}\left(p_{1}\right)-E\right)\left(E_{3}\left(p_{2}\right)+E_{4}\left(p_{2}\right)-E\right)}
$$

and the differential cross section with the final second channel is proportional to

$$
\frac{d \sigma}{d E} \sim \frac{p(E)}{|1-N(E)|^{2}}
$$

where $p(E) \sim \sqrt{E^{2}-\left(m_{3}+m_{4}\right)^{2}}$. It is interesting, that for the fully symmetric case, when all $\beta_{i}$ are equal, and $\omega_{1}=\Omega_{1}$, one obtains for the exponent in (3.13) $\exp \left(-\frac{p_{1}^{2}+p_{2}^{2}}{2 \beta^{2}}\right)$, and $V_{12 \mid 34}=V_{34 \mid 12}$ and $N(E)$ are

$$
\begin{aligned}
V_{12 \mid 34}^{\mathrm{symm}}\left(\mathbf{p}_{1}, \mathbf{p}_{2}\right) & =\frac{2^{5 / 2} \sqrt{\pi}}{\beta^{3}} \bar{y}_{1234} \exp \left(-\frac{p_{1}^{2}+p_{2}^{2}}{4 \beta^{2}}\right) \\
N(E) & =\frac{2 M_{\omega}^{2} \bar{\chi}_{1234}^{2}}{\pi \beta^{6}} \int \frac{p_{1}^{2} d p_{1} p_{2}^{2} d p_{2} \exp \left(-\frac{p_{1}^{2}+p_{2}^{2}}{4 \beta^{2}}\right)}{\left(E_{1}\left(p_{1}\right)+E_{2}\left(p_{1}\right)-E\right)\left(E_{3}\left(p_{2}\right)+E_{4}\left(p_{2}\right)-(E)\right.} .
\end{aligned}
$$

\section{Recoupling mechanism for the meson-meson amplitudes}

The formalism introduced on the previous section can be directly applied to the amplitudes, containing two meson-meson thresholds, $m_{1}+m_{2} \leftrightarrow m_{3}+m_{4}$ with the singularities given by the equation

$$
1-N\left(m_{1}, m_{2}, m_{3}, m_{4} ; E\right)=0 .
$$

As we discussed in section II, the conditions for the appearance of visible singularities require that the threshold difference $\Delta M=m_{3}+m_{4}-m_{1}-m_{2}$ should be comparable or smaller than average size $\langle\beta\rangle$ of the hadron wave functions in momentum space, while 
the recoupling coefficient $\bar{y}_{1234}^{2}$ is of the order of unity, i.e. there should be no angular momentum excitation or spin flip process.

An additional requirement is the relatively small widths of participating hadrons, otherwise all singularities would be smoothed out.

One can choose several examples in this respect.

1) The set of tranformations

$$
J / \psi+\phi \leftrightarrow D_{s}^{*}+\bar{D}_{s}^{*} \rightarrow J / \psi+\phi
$$

with masses $m_{1}=3097 \mathrm{MeV}, m_{2}=1020 \mathrm{MeV}, m_{3}=m_{4}=2112 \mathrm{MeV}$, and the corresponding thresholds $m_{1}+m_{2}=4117 \mathrm{MeV}$ and $m_{3}+m_{4}=4224 \mathrm{Mev}$. One can see no spin flip in the sequence $c_{+} \bar{c}_{+}+s_{+} \bar{s}_{-} \rightarrow c_{+} \bar{s}_{-}+\bar{c}_{+} s_{+}$for (4.1), where lower indices denote spin projections, and therefore no damping of transition probability. One can expect, that the yield of the reaction (4.2) would have the form similar to that of $\chi_{c 1}(4140)$ with the mass $(4147 \mathrm{MeV}$ with the width $\Gamma=(22 \pm 8) \mathrm{MeV}$ [51-54].

2) One of the best studied exotic resonances $Z_{c}(3900)$ [55-58] was found in the reaction $e^{+} e^{-} \rightarrow \pi^{+} \pi^{-} J / \psi \rightarrow \pi^{ \pm} Z_{c}(3900)$. It can be associated with the recoupling process $D \bar{D}^{*} \leftrightarrow \pi J / \psi$, where the higher threshold is $M_{2}=3874 \mathrm{MeV}$, and the spin, charge and isospin recombination agrees with this recoupling. One expects the peak above $M_{2}$ in agreement with experiment.

A similar situation can be in the case of the $Z_{c}(4020)$ observed in the reaction $e^{+} e^{-} \rightarrow$ $\pi \pi h_{c}$ [59], which can be associated with the recoupling $\pi h_{c} \rightarrow D^{*} \bar{D}^{*}$ with threshold $M_{1}=3665 \mathrm{MeV}$ and $M_{2}=4020 \mathrm{MeV}$. One can one can envisage the yield of the reaction to be described by the equation (3.18), with $p(E) \rightarrow p^{3}(E)$, since one need the $P$-wave in $D^{*} \bar{D}^{*}$ near threshold, as in $h_{c}$.

Note, that in general the recoupling can easily produce both $Z_{b}, Z_{c}$ resonance peaks, when a charged particle (like $\rho$ ) is participating in the sequence of transformations.

\section{Recoupling mechanism for meson-baryon systems}

One can consider the transformation sequence for baryons of the form, e.g.

$$
(q q q)+Q \bar{Q}) \leftrightarrow(q q Q)+(q \bar{Q})
$$

and apply the same formalism as the used above for the meson-meson recoupling transformations.

In principle it implies the new degrees of freedom, associated with the additional quark in $(q q q)$ as compared to the meson $(q \bar{q})$. To simplify the matter, we start below with the assumption, that the diquark combination can be factorized out in the baryon $(q q q) \rightarrow$ $q(q q)$ and does not change during the recoupling process, which can now be written as

$$
q(q q)+(Q \bar{Q}) \leftrightarrow Q(q q)+(q \bar{Q}) .
$$


In doing so we neglect also the internal structure of the diquark $(q q)$ system, which stays unchanged during the recoupling process, so that only its total spin, spin projection and its relative motion with the quark $q$ or $Q$ in the baryon bound state is present in the matrix element (3.11), while the norm of $(q q)$ is factored out. As a result one can use eqs. (3.11), (3.13), where we need the wave functions of the relative motion of quark and diquark in the baryons $q(q q)$ and $Q(q q)$. Using our notations $h_{1}\left(q_{1} \bar{Q}_{1}\right)+h_{2}\left(\bar{q}_{2} Q_{2}\right) \leftrightarrow h_{3}\left(q_{1} \bar{q}_{2}\right)+h_{4}\left(\bar{Q}_{1} Q_{2}\right)$ we are replacing $\bar{q}_{2}$ by the diquark $\left(q q^{\prime}\right)$. The accuracy of this replacement was discussed in literature [61-65] and the interactions are discussed and compared in [66]. In what follows we need the approximate baryon wave functions as $\psi_{2}(\mathbf{y}-\mathbf{v}) e^{-i \mathbf{p}_{1} \mathbf{R}(\mathbf{y}, \mathbf{v})}$ and $\psi_{3}(\mathbf{u}-\mathbf{v}) e^{-i \mathbf{p}_{2} \mathbf{R}(\mathbf{u}, \mathbf{v})}$ in (3.2), (3.4), where $\mathbf{v}$ denotes the center-of-mass of the diquark, and $\mathbf{R}(\mathbf{y}, \mathbf{v})$ is the c.m. of the quark-diquark combination, i.e. actually is the c.m. of the baryon $Q_{2}(q q)$. The same for the $\psi_{3}(\mathbf{u}-\mathbf{v})$ and its c.m. $\mathbf{R}(\mathbf{u}, \mathbf{v})$. Using the oscillator forms for $\psi_{2}, \psi_{3}$ one is actually exploiting the description of the only one part (factor) of the baryon wave function, which can be associated with only one leaf of the three-leaf baryon configuration. As a result, one can approximate this part of the wave function with the wave function of the heavy-light meson for the $Q_{2}(q q)$ baryon $\left(Q_{2}(q q) \rightarrow Q_{2} \bar{q}\right)$ or with the light baryon for the $h_{3}\left(q_{1} \bar{q}_{2} \rightarrow q_{1}(q q)\right)$.

As a first example one can take the transitions $p+\phi \rightarrow \bar{K}^{*}+\Lambda$ with thresholds $M_{1}=1960 \mathrm{MeV}$ and $M_{2}=2005 \mathrm{MeV}$, where the role of quarks $\bar{Q}_{1}, Q_{2}$ is played by $\bar{s}, s$ and one has a transition $u(u d)+\bar{s} s \rightarrow \bar{s} u+s(u d)$, where all $\beta$ parameters have a similar magnitude, and one can expect a peak nearby $M_{2}$.

As a concrete example one can take the case of the triad $P_{c}(4312), P_{c}(4440)$ and $P_{c}(4457)$, found experimentally in $[67,68]$, with a vast literature devoted to this phenomenon, called pentaquark, see a review in $\mathrm{PDG},{ }^{2}$ and for the latest pentaquark papers see $[69-86]$.

The most part of the literature considers pentaquarks as a result of molecular interaction between a white baryon and a white meson, which creates a bound state nearby the threshold of this system. In what follows we shall exploit the recoupling mechanism and we shall show, that it can provide the observed peaks without an assumption of the white-white strong interaction.

We shall have in mind the recoupling transformations of the type

$$
J / \psi+P \leftrightarrow\left(\Sigma, \Sigma^{*}\right)+\left(D, D^{*}\right)
$$

and we impose the requirement of $s$-wave recoupling without spin flip processes and parity conservation, which excludes $\Lambda_{c}^{*}(2595)$ with $\left(I J^{P}\right)=\left(0, \frac{1}{2}^{-}\right)$and includes $\Lambda_{c}(2286)\left(0, \frac{1}{2}^{+}\right)$, $\Sigma_{c}(2455)\left(1, \frac{1}{2}^{+}\right), \Gamma \approx 2 \mathrm{MeV}, \Sigma_{c}^{*}(2529)\left(1, \frac{3}{2}^{+}\right), \Gamma \approx 15 \mathrm{MeV}$, in addition to $D(1864),\left(\frac{1}{2}, 0^{+}\right)$ and $D^{*}(2010),\left(\frac{1}{2}, 1^{-}\right)$with $\Gamma_{D}, \Gamma_{D^{*}}<1 \mathrm{MeV}$. 


\begin{tabular}{|c|c|c|c|c|}
\hline thresholds $(\mathrm{MeV})$ & 4150 & 4319 & 4465 & 4384 \\
\hline pairs & $\Lambda_{c} \bar{D}$ & $\Sigma_{c} \bar{D}$ & $\Sigma_{c} \bar{D}^{*}$ & $\Sigma_{c}^{*} \bar{D}$ \\
\hline pentaquarks & & $P_{c}(4312)$ & $P_{c}(4457)$ & $P_{c}(4440)$ \\
width, $\mathrm{MeV}$ & & $\Gamma=9.8$ & $\Gamma=6.4$ & $\Gamma=20.6$ \\
\hline
\end{tabular}

Table 1. Meson-baryon thresholds and the associated pentaquarks.

As a result one obtains the thresholds $M_{2}=m_{3}+m_{4}$ in the table 1 together with $P_{c}$. Following the table, we can consider two types of reactions,

$$
\begin{aligned}
\text { I. } & (c(u d))\left(\Sigma_{c}, \Sigma_{c}^{*}\right)+(\bar{c} u)\left(\bar{D}, \bar{D}^{*}\right) & \leftrightarrow(c \bar{c})(J / \psi)+(u(u d))(p) \\
I I . & (c(u u))\left(\Sigma_{c}^{*}\right)+\bar{c} d\left(\bar{D}, \bar{D}^{*}\right) & \leftrightarrow(c \bar{c})+(u(u d))(p) .
\end{aligned}
$$

To proceed one needs the values of $\beta_{i}, i=1,2,3,4$ and $A, B, C$ and $a$ in (3.17). Using appendix A one finds the values of $\omega, \Omega$ in (3.14), (3.15) $\Omega_{1}=1509 \mathrm{MeV}, \omega_{1}=507 \mathrm{MeV}$. From appendix $\mathrm{C}$ one finds the values of $\beta_{i}$ :

$$
\beta_{i}\left(D, D^{*}\right) \cong \beta_{2}(\Sigma)=0.48 \mathrm{GeV}, \quad \beta_{3}(p) \approx 0.26 \mathrm{GeV}
$$

and $\beta_{4}(J / \psi)=0.7 \mathrm{GeV}$. Note, that for $\Sigma$ and $p$ we have used the principle of replacement of light diquark by a light antiquark, $(u d) \rightarrow \bar{q}$. Therefore $\beta(\sigma)=\beta(c(u d)=\beta(c \bar{u})=\beta(D)$.

As a consequence one obtains the values given in (5.6). Using those we get the numerical values for $a, A, B, C$.

$$
a=12.76 \mathrm{GeV}^{-2}, \quad A=4.02 \mathrm{GeV}^{-2}, B=0.94 \mathrm{GeV}^{-2}, \quad|C|<0.03 \mathrm{GeV}^{-2} .
$$

As a result one can neglect the $C \mathbf{p}_{1} \mathbf{p}_{2}$ in (3.17) and the integrals $d^{3} \mathbf{p}_{1}, d^{3} \mathbf{p}_{2}$ factorize.

We turn now to the recoupling coefficients $M_{\omega}, \bar{y}_{1234}$.

As it was shown in [50], the effective parameter $M_{\omega}$ can be expressed via the wave functions of objects, produced by the string breaking, in our case it is heavy-light mesons with the coefficient $\beta(D) \cong \beta(B)=0.48 \mathrm{GeV}$, and from eq. (35) of [50] one has

$$
M_{\omega} \cong \frac{2 \sigma}{\beta(D)} \cong 0.8 \mathrm{GeV}
$$

Finally, the coefficient $\bar{\chi}_{1234}$ for the transition into $\left(\Sigma_{c} \bar{D}\right)$ and $\left(\Sigma_{c} \bar{D}^{*}\right)$ can be estimated as in the appendix B to be equal to 1.

\section{Physical amplitudes and their singularities in the recoupling process}

As was discussed in section 3, (3.18), the differential cross section for the production of hadrons in channel 1 can be written as

$$
\frac{d \sigma}{d E}=\left|F_{1}(E) f_{12}(E)\right|^{2} p_{1}(E)
$$


where $F_{1}(E)$ is the production amplitude of channel 1 particles without final state $F S$ interaction and $f_{12}$ is the $F S$ interaction, which we take as an infinite sum of transitions from channel 1 to channel 2-the Cornell-type mechanism [30-35].

$f_{12}(E)$ can be written as

$$
f(E)=\frac{1}{1-N(E)}
$$

where $N(E)$ has the form

$$
N(E)=\lambda I_{1}(E) I_{2}(E),
$$

where $I_{i}(E)$ has the form

$$
I_{i}(E)=\int \frac{d^{3} p_{i}}{(2 \pi)^{3}} \frac{v_{i}^{2}\left(p_{i}\right)}{E^{\prime}\left(p_{i}\right)+E^{\prime \prime}\left(p_{i}\right)-E} .
$$

Here $v_{i}$ is proportional to the product of wave functions in momentum space (see (ref 42)) and can be written in two forms: a/ as a Gaussian of $p$ and b/ as an inverse of $\left(p^{2}+\nu^{2}\right)$. To simplify matter we shall consider situation close to nonrelativistic for the energies in the denominator of (6.4) and write

$$
E^{\prime}\left(p_{1}\right)+E^{\prime \prime}\left(p_{1}\right)=m_{1}+m_{2}+\frac{p_{1}^{2}}{2 \mu_{1}}, E^{\prime}\left(p_{2}\right)+E^{\prime \prime}\left(p_{2}\right)=m_{3}+m_{4}+\frac{p_{2}^{2}}{2 \mu_{2}} .
$$

We have considered above in the paper $v_{i}^{2}(p)$ as a Gaussian $\exp \left(-b_{i} p_{i}^{2}\right.$ with $b_{1}=2 B, b_{2}=$ $2 A$, see (3.17). We simplify below this expression, writing $\exp \left(-b_{i} p^{2}\right)=\frac{1}{\exp \left(b_{i} p^{2}\right)} \approx$ $b_{i}^{-1} \frac{1}{p^{2}+\nu_{i}^{2}}$, where $\nu_{i}=\frac{1}{\sqrt{b_{i}}}$. As a result one can write for $I_{i}(E)$ in the region $E>E_{i}(t h)=$ $m_{1}+m_{2}(i=1)$ or $m_{3}+m_{4}(i=2)$

$$
I_{i}(E)=\frac{\mu_{i}}{2 \pi b_{i}} \nu_{i}-i \sqrt{2 \mu_{i} \Delta_{i}}, \Delta_{i}=E-E_{i}(t h)
$$

As a result one obtains a simple expression for the amplitude $f_{12}(E)$

$$
f_{12}(E)=\frac{1}{1-\frac{\lambda^{\prime} \mu_{1} \mu_{2}}{\left(\nu_{1}-i \sqrt{2 \mu_{1} \Delta_{1}}\right)\left(\nu_{2}-i \sqrt{\left.2 \mu_{2} \Delta_{2}\right)}\right.}}=\frac{1}{1-z t_{1}(E) t_{2}(E)} .
$$

Here $z=\frac{\lambda}{\mu_{1} \mu_{2} b_{1} b_{2}(2 \pi)^{2}}$ and the (6.7) refers to the region $E>E_{1}($ th $), E_{2}($ th) otherwise one should replace $-i$ before the $\sqrt{ }$ terms by $(+1)$ for the roots, where $\Delta(E)$ is negative.

We now consider the channel coupling constant $\lambda^{\prime}$, which enters (6.7). From (3.17) one obtains

$$
\lambda^{\prime}=\frac{M_{\omega}^{2} \chi_{1234}^{2} b_{1} b_{2}}{4 \pi^{5} a^{3}\left(\prod_{i} \beta_{i}\right)^{3}} .
$$

The resulting $z$ is larger than unity for the recoupling coefficient $\chi_{1234}$ of the order of 1 , and one can vary $z$ in the interval from one to larger values. At this point we shall discuss the physical structure of the obtained expression for the amplitude $f_{12}(E)(6.7)$. One can see that $f_{12}$ contains the product of two unitary single channel amplitudes $t_{1}, t_{2}$ which describe separately scattering amplitudes in channel 1 and 2 characterizing by numbers $\nu_{i}, i=1,2$. The latter are obtained from the hadron wave functions and reflect their 
structure given by the Gaussian coefficients $\beta_{i}$. Now let us study the structure of the possible singularities of $f_{12}$ in the $E$ plane, which is given by zeros of the denominator of $f_{12}$ in (6.7) and we concentrate on the $k_{1}$ plane while the second channel is characterized by the value of $B(E)=\frac{1}{\nu_{2}-i k_{2}}$ and $-i k_{2}>0$ below higher threshold $E_{2}$. As a result one obtains an equation for the pole of $f_{12}$

$$
k_{1}=-i \nu_{1}+i z B(E), B(E)>0, \nu_{1}>0 .
$$

It is evident that originally (at $z B=0$ ) virtual pole of $k_{1}$ is proceeding to the upper half plane of $k_{1}$ with increasing $z B$, and at some $z B$ at appears at $k_{1}=0$ i.e. at the threshold $E_{1}$, and at larger $z B$ one obtains a real pole, i.e. the effect of recoupling with the channel 2 increases the attraction and creates a real pole from the original distant virtual pole. However for the physical amplitude $t_{1}(E)$ of the hadron-hadron interaction the virtual pole can be not a good approximation and more generally one should write for the amplitude $t_{1}(E)=\frac{1}{\nu_{1}(E)-i k_{1}}$ the $\nu_{1}(E)$ in the form $\nu_{1}(E)=a+b \ln \left(\frac{m^{2}+4 k_{1}^{2}}{m^{2}}\right)$, where $a, b$ are positive numbers. This construction occurs from the t-channel exchange of the hadron with mass $m$. One can see from (6.9) that the logarithmic singularity is moving towards the threshold with the increasing $z$ and can appear on the first sheet $\left(E<E_{1}\right)$. We can now study the situation with the singularities associated with the threshold $E_{2}$ and find that here the situation is completely different because when $E E_{2}$ the momentum $k_{1}(E)$ as a rule is large and positive imaginary. As a result there is no pole solutions of the equation (6.7) for all values of $E$ nearby the threshold $E_{2}$ and all resulting widths are of the order of parameters $\mu_{i}, \nu_{i}$, i.e. $(0.5-1) \mathrm{GeV}$, and therefore cannot be seen in experiment. Nevertheless we give in the Apendix 4 full quartic equation and its analysis for the finding of resonance poles in the whole combination of $k_{1}, k_{2}$ planes. As a special case one can consider the situation when the distance $\Delta=E_{2}-E_{1}$ is small as compared with $\nu_{i}, i=1,2$. In this case the amplitude $f_{12}(E)$ written as

$$
f_{12}=\frac{k_{2}-i \nu_{2}}{k_{2}+\frac{z k_{1}}{\nu_{1}^{2}+k_{1}^{2}}+i\left(\nu_{2}-\frac{z \nu_{1}}{\nu_{1}^{2}+k_{1}^{2}}\right)} .
$$

One can see that for $k_{1} \ll \nu_{1}$ and $\nu_{2} \frac{z}{\nu_{1}}$ one obtains a resonance pole slightly shifted above the threshold $E_{2}$, however this situation is coincidental. Summarizing we expect that the resulting singularities of the recoupling amplitude are produced by the singularities of the lower threshold process (process 1 in our definition) shifted to the lower threshold due to recoupling interaction with the process 2 ). In the next section we shall study this effect numerically in real physical examples.

\section{Numerical results and discussion}

We can now consider 3 transitions, partly discussed above:

1)

$$
\begin{aligned}
& J / \psi+\phi \rightarrow D_{s}^{*}+\bar{D}_{s}^{*} \\
& E_{1}(\mathrm{th})=4.12 \mathrm{GeV}, E_{2}(\mathrm{th})=4.224 \mathrm{GeV}, \mu_{1}=0.767, \mu_{2}=1.056, \nu_{1}=0.96, \nu_{2}= \\
& 0.87, z=1 \text { (all GeV). }
\end{aligned}
$$




\begin{tabular}{|c|c|c|c|c|c|c|}
\hline$E(\mathrm{GeV})$ & 4.0 & 4.05 & 4.12 & 4.17 & 4.224 & 4.3 \\
$\left|f_{12}(E)\right|^{2}$ & 3.43 & 4.49 & 17.36 & 10.76 & 5.6 & 1.24 \\
\hline
\end{tabular}

Table 2. The values of the $\left|f_{12}(E)\right|^{2}$ near the channel thresholds for the transition 1).

2)

$$
\begin{gathered}
J / \psi+\phi \rightarrow D_{s}+\bar{D}_{s} \\
E_{1}(\mathrm{th})=3.936, E_{2}(\mathrm{th})=4.12, \mu_{1}=0.767, \mu_{2}=0.984, \nu_{1}=0.96, \nu_{2}=0.87, z=1 .
\end{gathered}
$$

As a special interesting case we consider below the recent experiment of BES III [87],

$$
e^{+}+e^{-} \rightarrow K^{+}\left(D_{s}^{-} D^{*}+D_{s}^{*-} D\right) .
$$

Applying here our recoupling mechanism, shown in the figure 1, one easily finds that the second channel obtained from the first channel $D_{s}^{-} D^{*}$ by recoupling is the channel $J / \psi+K^{*-}$ which creates the chain of reactions possibly generating a peak in the system $D_{s}^{-} D^{*}$ or $D_{s}^{*-} D$, namely we consider as the third example

3)

$$
\begin{aligned}
& D_{s}^{-}+D_{s}^{*} \rightarrow J / \psi+K^{*-} \\
& E_{1}(\mathrm{th})=3.975, E_{2}(\mathrm{th})=3.992, \mu_{1}=0.9936, \mu_{2}=0.692, \nu_{1}=0.87, \nu_{2}=0.96 \text { (all } \\
& \mathrm{GeV}) .
\end{aligned}
$$

One can easily find that in all cases the values of $\nu_{i}$ and the values of $\mu_{i}$ are in the range $0.35-1.06 \mathrm{GeV}$. Applying the (6.7) one can find the $f_{12}$ in all cases and hence $\frac{d \sigma}{d E}$ in the cases 1), 2), 3), assuming $z$ as a positive number typically larger or equal 1 . However for the 3) one could use also the sum: $f_{12} \rightarrow f_{12}+\alpha f_{12}^{\prime}$, implying possible superposition of intermediate states in the rescattering series.

We proceed now with the cases 1)-3) and insert the values of $E_{i}(t h), \mu_{i}, \nu_{i}$ in (6.3), (6.7) and fixing the value of $z$ one obtains the form of the recoupling amplitude shown in the tables $2-4$ below.

For the case 1) the resulting values of $\left|f_{12}(E)\right|^{2}$ can be seen in the table 2 .

One can see in the table 2 a strong enhancement around $E=4.12 \mathrm{GeV}$ with the width around $10 \mathrm{MeV}$ which can be associated with the resonance $\chi_{c 1}(4140)$ having the mass $4147 \mathrm{MeV}$ and the width $\Gamma=22 \mathrm{MeV}$.

It is now interesting how these numerical data are explained by the exact solution of the equation (6.7), (6.9). From these equations one obtains the exact position of the pole $k_{1}=i 0.068 \mathrm{GeV}, E=E_{1}-0.003 \mathrm{GeV}$ which is unphysical result due to too small values of $\nu_{1}$, and in a more realistic case of the virtual pole its position moves to $k_{1}=0, E=E_{1}$ for $\nu_{1}=0.84 \mathrm{GeV}$. Three more complex poles are $\mathrm{O}(1 \mathrm{GeV})$ far from the thresholds, as can be found from the solution of the quartic equation in appendix D. Looking at table 2 one can see a good agreement with this result. In a similar way we obtain the results for the $J / \psi+p$ transitions of $\mathbf{2}$ ) 


\begin{tabular}{|c|c|c|c|c|c|c|c|}
\hline$E(\mathrm{GeV})$ & 3.8 & 3.85 & 3.936 & 4.0 & 4.05 & 4.12 & 4.2 \\
$\left|f_{12}(E)\right|^{2}$ & 3.12 & 3.84 & 18.9 & 6.15 & 5.33 & 298 & 1.17 \\
\hline
\end{tabular}

Table 3. The values of the $\left|f_{12}(E)\right|^{2}$ near the $\Sigma D$ threshold.

\begin{tabular}{|c|c|c|c|c|c|c|}
\hline$E(\mathrm{GeV})$ & 3.96 & 3.975 & 3.98 & 3.985 & 3.992 & 4.0 \\
$\left|f_{12}\right|^{2}(z=1)$ & 5.43 & 322 & 67.56 & 31.84 & 12.95 & 5.54 \\
$\left|f_{12}\right|^{2}(z=1.5)$ & 19.9 & 2.93 & 3.19 & 2.58 & 1.61 & 2.71 \\
\hline
\end{tabular}

Table 4. The values of the transition probability as a function of energy in the transition $\mathbf{3}$ ).

One can see in table 3 a strong peak near the lower threshold $E=3.94 \mathrm{GeV}$ and it is easy to check that this peak is stable when one varies $z$ in the region around $z=1$, One can associate this peak with the virtual pole appearing at $50 \mathrm{MeV}$ below the threshold $E_{1}=3.94 \mathrm{GeV}$. The exact form of the solution for $f_{12}(E)$ near the peak has the form $f_{12}(E)=\frac{k_{1}+i 0.96}{k_{1}+i 0.28}$. In this way our method can support the origin of the $X(3915)$ state as due to the $J / \psi+\phi \leftrightarrow D_{s}+\bar{D}_{s}$ transitions.

We come now to the recent interesting discovery of the new state $Z_{c s}(3985)$ [87], where we take for simplicity only the first chain denoted as the $\mathbf{3}$ ) above. Similarly to the previous cases one obtains

One can see in table 4 a narrow peak with the summit at $E=3.975 \mathrm{GeV}$ for $z=1$ with the width around $10 \mathrm{MeV}$, which closely corresponds to the experimental data from [87] $E=$ $3.982 .5, \Gamma=12.8 \mathrm{MeV}$. In our case the resonance parameters weakly depend on $z$. As seen in the table 4 , for $z=1.5$ the peak shifts down to $E=3.960 \mathrm{GeV}$ with much larger width. Its form is exactly the same as in the previous two examples and corresponds to virtual pole almost exactly at the lower threshold. In this way we can explain the newly discovered resonance $Z_{c s}(3985)$ by the recoupling mechanism in the rescattering series of transitions

$$
D_{s}^{-}+D_{s}^{*} \rightarrow J / \psi+K^{*-} .
$$

\section{Conclusions and an outlook}

As it was shown above, the new mechanism having the only parameter $z$ is able to predict and explain the resonances in different systems, as it was shown above, and possibly in other systems which can transfer one into another via the recoupling of the confining strings. The necessary conditions for the realization of these transitions and the appearance of a resonance are connected to the value of the transition coefficient $z$, which should be of the order of unity or larger. Therefore the transition should be strong, i.e. without serious restructuring of the hadrons involved, since otherwise the transition will be strongly suppressed e.g. in the case when not only strings are recoupled, but also spins,orbital momenta, isospins should be exchanged. In any case the suggested mechanism provides an alternative to the popular tetra - and pentaquark mechanisms, which dominate in the literature. One should stress at this point, that the independent and objective checks, e.g. 
the lattice calculations do not give strong support for the molecular or tetraquark models and the existence of an independent mechanism is welcome. It is necessary that this method should be studied more carefully. As to the recoupling mechanism, it is strongly associated with the thresholds participating in the transitions, and the best situation for its application is when both thresholds are close by. In all 3 cases considered above the distance between threshold was less than $200 \mathrm{MeV}$, and in all cases one could see a strong enhancement in the transition coefficient and hence in the resulting cross section. The necessary improvements of the present study are 1) a more accurate calculation of the coefficient $z$ (originally $\lambda^{\prime}$ ), and 2) the use of a more realistic Gaussian approximation for the wave functions instead of approximate $\nu_{i}$ parametrizations to define $f_{12}$ with good accuracy in the future.

\section{Acknowledgments}

The author is grateful to Lu Meng for an important remark, to R.A.Abramchuk for useful discussians and to A.M.Badalian for discussions and suggestions. This work was done in the frame of the scientific project supported by the Russian Science Foundation Grant No. 16-12-10414.

\section{A The center-of-mass coordinates and average quark and antiquark en- ergies in a hadron}

Following [49] one can define the c.m. coordinate of a hadron consisting of a quark $Q$ at the point $\mathbf{x}$ and an antiquark $\bar{q}$ at the point $\mathbf{u}$ via average energies $\Omega$ and $\omega$ of $Q$ and $\bar{q}$ correspondingly as

$$
\mathbf{R}_{Q \bar{q}}=\frac{\Omega \mathbf{x}+\omega \mathbf{u}}{\Omega+\omega}, \quad \Omega=\left\langle\sqrt{\mathbf{p}_{Q}^{2}+m_{Q}^{2}}\right\rangle, \quad \omega=\left\langle\sqrt{\mathbf{p}_{q}^{2}+m_{q}^{2}}\right\rangle
$$

where $\sqrt{\mathbf{p}_{Q}^{2}+m_{Q}^{2}}+\sqrt{\mathbf{p}_{q}^{2}+m_{q}^{2}}$ is the kinetic part of the $Q \bar{q}$ Hamiltonian in the so-called spinless Salpeter formalism or an equivalent form in the so-called einbein formalism. ${ }^{3}$

As a result one obtains the following value of $\omega=\Omega$ for $q \bar{q}$ mesons, shown in table 2 .

\section{B The channel-coupling coefficient $\bar{y}_{1234}$}

We discuss here two topics: 1) the problem of the double string decay vertex contribution to the recoupling coefficient $M_{\omega}$ in $\left.(3.11), 2\right)$ the construction of the recoupling vertex $\bar{y}_{1234}$.

We start with the topic 1), and following [50] define the relativistic expression for the string decay vertex as in (3.9), but without free parameters namely $M(x)$ in (3.9) is

$$
M(x)=\sigma\left(\left|\mathbf{x}-\mathbf{x}_{Q}\right|+\left|\mathbf{x}-\mathbf{x}_{\bar{Q}}\right|\right) .
$$

As one can see in figure 2, in our case the structure of the recoupling process can be explained by the double string breaking, which we can write as a product

$$
S=\int d^{4} x \bar{\psi}(x) \bar{M}(x) d^{4} y \psi(x) \bar{\psi}(y) \bar{M}(y) \psi(y)
$$

\footnotetext{
${ }^{3}$ See a short recent review in the last refs. in [44-46].
} 


\begin{tabular}{|c|c|c|c|c|c|}
\hline State & $J / \psi$ & $\psi(2 S)$ & $\psi(3770)$ & $\psi(3 S)$ & $\psi(4 S)$ \\
\hline$\Omega, \mathrm{GeV}$ & 1.58 & 1.647 & 1.640 & 1.711 & 1.17 \\
\hline State & $\Upsilon(1 S)$ & $\Upsilon(2 S)$ & $\Upsilon(3 S)$ & $\Upsilon(4 S)$ & $\Upsilon(5 S)$ \\
\hline$\Omega, \mathrm{GeV}$ & 5.021 & 5.026 & 5.056 & 5.088 & 5.120 \\
\hline Sate & $D$ & $D_{s}$ & $B$ & $B_{s}$ & $\rho$ \\
\hline$\Omega, \mathrm{GeV}$ & 1.509 & 1.515 & 4.827 & 4.830 & 0.4 \\
\hline$\omega, \mathrm{GeV}$ & 0.507 & 0.559 & 0.587 & 0.639 & 0.4 \\
\hline
\end{tabular}

*) Note, that the difference in $\Omega, \omega$ obtained in these approaches is less or around $1 \%$.

Table 5. Average values of quark and antiquark kinetic energies in different mesons.

and one must take into account, that the energy minimum of the resulting broken string occurs when both time moments $x_{0}, y_{0}$ of string breaking are equal. Indeed, taking the integral in (B.2) with account of the string action in the exponent of the path integral,

$$
\Delta S_{\text {string }}=\int \sigma \sqrt{r_{x y}^{2}+\left(x_{4}-y_{4}\right)^{2}} d \frac{x_{4}+y_{4}}{2},
$$

which produces a factor on (B.2) $\left\langle r_{x y}\right\rangle \sqrt{2 \pi}$, which denoted as $M_{\omega}$ in (3.11). The resulting double string breaking action can be written as,

$$
S \cong \int d^{3} x \bar{p} \operatorname{si}(x) \psi(x) d^{3} y \bar{\psi}(y) \psi(y) M_{\omega}(\mathbf{x}, \mathbf{y}) \frac{d\left(x_{4}+y_{4}\right)}{2}
$$

using the notation

$$
\int d\left(x_{4}-y_{4}\right)\langle\bar{M}(x) \bar{M}(y)\rangle=M_{\omega}(\mathbf{x}, \mathbf{y}) .
$$

In what follows one can estimate $\left\langle M_{\omega}(\mathbf{x}, \mathbf{y})\right\rangle \equiv M_{\omega}$ in the same way, as it was done in [50], with the result $M_{\omega} \approx \frac{2 \sigma}{\beta}$, where $\beta$ is the oscillator parameter for the $(Q \bar{Q})$ meson. We now turn to the point 2 ) above, the recoupling vertex $\bar{y}_{1234}$.

To define $\bar{y}_{1234}$ we notice that all 4 quarks $q, \bar{q}, Q, \bar{Q}$ keep their identity and spin polarization during the whole process of transformations, provided we neglect the spin dependent corrections. This can be also seen in the structure of the recoupling process: in (B.2) one does not see spin dependence, and this means, that the spin projection of each quark or antiquark is kept unchanged during recoupling. As a result one can write the nonrelativistic spin part of the matrix element $V_{12 / 34}$ as

$$
V_{\text {meson }}^{\text {spin }}=C_{\mu_{1} \mu_{2}}^{L m} \chi_{\mu_{1}}^{(1)} \bar{\chi}_{\mu_{2}}^{(2)} C_{\mu_{1}^{\prime} \mu_{2}^{\prime}}^{L^{\prime} m^{\prime}} \chi_{\mu_{1}^{\prime}}^{(3)} \bar{\chi}_{\mu_{2}^{\prime}}^{(4)}\left(C_{\nu_{1} \nu_{2}}^{J M} \chi_{\nu_{1}}^{(5)} \bar{\chi}_{\nu_{2}}^{(6)} C_{\nu_{1}^{\prime} \nu_{2}^{\prime}}^{J^{\prime} M^{\prime}} \chi_{\nu_{1}^{\prime}}^{(7)} \bar{\chi}_{\nu_{2}^{\prime}}^{(8)}\right)
$$

where the Klebsch-Gordon coefficient $C_{\nu_{1} \nu_{2}}^{J M} \equiv C_{\frac{1}{2} \nu_{1} \frac{1}{2} \nu_{2}}$ and $\chi_{\mu}^{(i)}, \bar{\chi}_{\lambda}^{(k)}$ are quark and antiquark spinors.

As was told above, due to the spin conservation in recoupling, the matrix element (B.4) should be proportional to $\delta_{26} \delta_{48} \delta_{17} \delta_{35}$, implying the recoupling of quarks. 


\begin{tabular}{|c|c|c|c|c|c|}
\hline State & $\Upsilon(1 S)$ & $\Upsilon(2 S)$ & $\Upsilon(3 S)$ & $\Upsilon(4 S)$ & $\Upsilon(5 S)$ \\
\hline$\beta, \mathrm{GeV}$ & 1.27 & 0.88 & 0.76 & 0.64 & 0.6 \\
\hline State & $J / \psi$ & $\psi(2 S)$ & $\psi(3 S)$ & $\psi(4 S)$ & $\psi(5 S)$ \\
\hline$\beta, \mathrm{GeV}$ & 0.7 & 0.53 & 0.48 & 0.43 & 0.41 \\
\hline Sate & $D$ & $B$ & $\rho$ & & \\
\hline$\beta, \mathrm{GeV}$ & 0.48 & 0.49 & 0.26 & & \\
\hline
\end{tabular}

Table 6. The Gaussian parameters $\beta$ of different mesons.

As a result one obtains

$$
V^{\text {spin }}=\sum_{\mu_{i}, \mu_{k}^{\prime}} C_{\mu_{1} \mu_{2}}^{L m} C_{\mu_{1}^{\prime} \mu_{2}^{\prime}}^{L^{\prime} m^{\prime}} C_{\mu_{1}^{\prime} \mu_{2}}^{J M} C_{\mu_{1} \mu_{2}^{\prime}}^{J^{\prime} M^{\prime}}
$$

Here $L, L^{\prime}, J, J^{\prime}$ correspond to the spin values of hadrons $L+L^{\prime} \rightarrow J+J^{\prime}$ and we have assumed zero orbital momenta for all hadrons. Finally for the final expression in (3.11), $\left(V^{\text {spin }}\right)^{2}$ should be summed up over all $m, m^{\prime} M, M^{\prime}$, so that for $\bar{y}_{1234}^{2}$ one has

$$
\left\langle\left(\bar{y}_{1234}\right)^{2}\right\rangle=\sum_{m, m^{\prime} M, M^{\prime}}\left(V^{\text {spin }}\right)^{2} .
$$

In a similar way one can find the recoupling coefficient $\bar{y}_{1234}$ for the ensemble transformation

$$
p+J / \psi \rightarrow \Sigma\left(\Sigma^{*}\right)+D\left(D^{*}\right) \rightarrow p+J / \psi .
$$

In this case we write the baryon wave function as $\psi_{B}=u(u d)_{0}$, where the lower indices imply the total spin of the diquark $(u d)$. In the simplest approximation one can approximate the proton as the quark-diquark combination $p=u(u d) \cong u \tilde{d}$, with the diquark $\tilde{d}$ kept unchanged $d$ during recoupling.

\section{Oscillator parameters of hadron wave function}

The oscillator parameters for the bottomonium, charmonium and $B, D$ mesons have been obtained in [37-46], using the expansion of relativistic wave functions, obtained from the solutions of the relativistic string Hamiltonian [49], in the full set of the oscillator wave functions. As a result one obtains

The accuracy of the oscillator one-term approximation can be judged by the relative value of the sum od squared coefficients of four higher term of expansion as compared to the square of the main term. This amounts to the accuracy of the order or less than $10 \%$ for lowest states of charmonia and bottomonia and few percent for $D, B, \rho$.

\section{Calculation of the cross sections $\left|f_{i k}\right|^{2}$ and pole positions}

As it is written in (6.7) the cross section is defined as $\frac{d \sigma}{d E}=\left|f_{i k}\right|^{2}$ where $f_{i k}=\frac{1}{1-z A B}$, and $\mathrm{z}$ is numerical parameter proportional to $\lambda^{\prime}$, where for three numerical examples $(1),(2),(3)$ 
in that section $z=1,5,1$ and $A=\frac{1}{\nu_{1}-i k_{1}}$ and $B=\frac{1}{\nu_{2}-i k_{2}}$. Moreover $k_{i}=\sqrt{2 \mu_{i}\left(E-E_{i}\right)}$ and parameters $\mu_{i}, \nu_{i}, E_{i}$ are given in the section. As it is seen in tables 2, 3, 4 the cross sections have peaks and our purpose here is to calculate the positions of the poles both in E plane and in $k_{i}$ plane. To do this one starts with two equations

$$
\left(\nu_{1}-i k_{1}\right)\left(\nu_{2}-i k_{2}\right)=z, \frac{k_{1}^{2}}{2 \mu_{1}}-\frac{k_{2}^{2}}{2 \mu_{2}}=E_{2}-E_{1} .
$$

One of possible strategy is to insert $k_{1}$ from the first equation into second equation and one obtains an equation of 4 -th power for $k_{2}$. Note that both $k_{i}$ are positive on the real axis for $E>E_{i}$ and positive imaginary below $E_{i}$. Among many roots of eq. we need the closest to the real axis. The pole positions are below in the table. Now defining from the first equation in (D.1) $k_{1}$ via $k_{2}$ and submitting it into second equation one obtains the quartic equation for $x=k_{2}+i \nu_{2}$

$$
x^{4}+a x^{3}+b x^{2}+c x+d=0,
$$

where the coefficients are

$$
a=-2 i \nu_{2}, b=2 \mu_{2} \Delta-\nu_{2}^{2}+\frac{\mu_{2} \nu_{1}^{2}}{\mu_{1}}, c=-2 i \frac{\nu_{1} \mu_{2} z}{\mu_{1}}, d=-\frac{m u_{2} z^{2}}{\mu_{1}} .
$$

Now taking values of these coefficients from itemi), for three transitions $i=1,2,3$ discussed in section Numerical examples and discussion with $z=1,0.2,1$ respectively for these transitions, and $\Delta=E_{2}-E_{1}$, one obtains the roots of this equation together with the corresponding values of the resonance energy. The results of this computation for the examples 1)-3) are given in the text of the section 7 .

Open Access. This article is distributed under the terms of the Creative Commons Attribution License (CC-BY 4.0), which permits any use, distribution and reproduction in any medium, provided the original author(s) and source are credited.

\section{References}

[1] Particle Data Group collaboration, Review of particle physics, Phys. Rev. D 98 (2018) 030001 [inSPIRE].

[2] N. Brambilla et al., The $X Y Z$ states: experimental and theoretical status and perspectives, Phys. Rept. 873 (2020) 1 [arXiv: 1907.07583] [inSPIRE].

[3] J.L. Rosner, Threshold effect and $\pi^{ \pm} \psi(2 S)$ peak, Phys. Rev. D 76 (2007) 114002 [arXiv: 0708.3496] [INSPIRE].

[4] E. Braaten and M. Lu, Line shapes of the Z(4430), Phys. Rev. D 79 (2009) 051503 [arXiv:0712.3885] [INSPIRE].

[5] K.-M. Cheung, W.-Y. Keung and T.-C. Yuan, Bottomed analog of $Z^{+}$(4433), Phys. Rev. D 76 (2007) 117501 [arXiv:0709.1312] [INSPIRE].

[6] C. Meng and K.-T. Chao, $Z^{+}(4430)$ as a resonance in the $D_{1}\left(D_{1}^{\prime}\right) D *$ channel, arXiv:0708.4222 [INSPIRE]. 
[7] G.-J. Ding, Understanding the charged meson Z(4430), arXiv:0711.1485 [INSPIRE].

[8] G.-J. Ding, W. Huang, J.-F. Liu and M.-L. Yan, $Z^{+}(4430)$ and analogous heavy flavor molecules, Phys. Rev. D 79 (2009) 034026 [arXiv:0805.3822] [INSPIRE].

[9] Y. Li, C.-D. Lu and W. Wang, Partners of Z(4430) and productions in B decays, Phys. Rev. D 77 (2008) 054001 [arXiv:0711.0497] [InSPIRE].

[10] L. Maiani, A.D. Polosa and V. Riquer, The charged Z(4433): towards a new spectroscopy, arXiv:0708.3997 [INSPIRE].

[11] C.-F. Qiao, A uniform description of the states recently observed at B-factories, J. Phys. G 35 (2008) 075008 [arXiv:0709.4066] [InSPIRE].

[12] X.-H. Liu, Q. Zhao and F.E. Close, Search for tetraquark candidate Z(4430) in meson photoproduction, Phys. Rev. D 77 (2008) 094005 [arXiv: 0802.2648] [INSPIRE].

[13] X. Liu, B. Zhang and S.-L. Zhu, The two-body open charm decays of $Z^{+}(4430)$, Phys. Rev. D 77 (2008) 114021 [arXiv:0803.4270] [INSPIRE].

[14] L. Maiani, A.D. Polosa and V. Riquer, The charged Z(4430) in the diquark-antidiquark picture, New J. Phys. 10 (2008) 073004 [INSPIRE].

[15] D.V. Bugg, How resonances can synchronise with thresholds, J. Phys. G 35 (2008) 075005 [arXiv: 0802.0934] [INSPIRE].

[16] T. Matsuki, T. Morii and K. Sudoh, Is the $Z^{+}(4430)$ a radially excited state of $D_{s}$ ?, Phys. Lett. B 669 (2008) 156 [arXiv:0805.2442] [INSPIRE].

[17] M. Cardoso and P. Bicudo, Microscopic calculation of the decay of Jaffe-Wilczek tetraquarks, and the Z(4433), AIP Conf. Proc. 1030 (2008) 352 [arXiv:0805.2260] [INSPIRE].

[18] Y.-R. Liu and Z.-Y. Zhang, A chiral quark model study of $Z^{+}(4430)$ in the molecular picture, arXiv:0908.1734 [INSPIRE].

[19] T. Branz, T. Gutsche and V.E. Lyubovitskij, Hidden-charm and radiative decays of the $Z(4430)$ as a hadronic $D_{1} \bar{D} *$ bound state, Phys. Rev. D 82 (2010) 054025 [arXiv: 1005.3168] [INSPIRE].

[20] G. Galata, Photoproduction of Z(4430) through mesonic Regge trajectories exchange, Phys. Rev. C 83 (2011) 065203 [arXiv:1102.2070] [inSPIRE].

[21] M. Nielsen and F.S. Navarra, Charged exotic charmonium states, Mod. Phys. Lett. A 29 (2014) 1430005 [arXiv:1401.2913] [INSPIRE].

[22] X.-W. Kang, Z.-H. Guo and J.A. Oller, General considerations on the nature of $Z_{b}(10610)$ and $Z_{b}(10650)$ from their pole positions, Phys. Rev. D 94 (2016) 014012 [arXiv: 1603.05546] [INSPIRE].

[23] F.K. Guo et al., Interplay of quark and meson degrees of freedom in near-threshold states: a practical parametrization for line shapes, Phys. Rev. D 93 (2016) 074031 [arXiv: 1602.00940] [INSPIRE].

[24] C. Deng, J. Ping, H. Huang and F. Wang, Systematic study of $Z_{c}^{+}$family from a multiquark color flux-tube model, Phys. Rev. D 92 (2015) 034027 [arXiv: 1507.06408] [InSPIRE].

[25] F.-K. Guo, C. Hanhart, U.-G. Meißner, Q. Wang, Q. Zhao and B.-S. Zou, Hadronic molecules, Rev. Mod. Phys. 90 (2018) 015004 [arXiv:1705.00141] [InSPIRE]. 
[26] Y.-R. Liu, H.-X. Chen, W. Chen, X. Liu and S.-L. Zhu, Pentaquark and tetraquark states, Prog. Part. Nucl. Phys. 107 (2019) 237 [arXiv: 1903.11976] [InSPIRE].

[27] Y.S. Kalashnikova and A.V. Nefediev, $X(3872)$ in the molecular model, Phys. Usp. 62 (2019) 568 [Usp. Fiz. Nauk 189 (2019) 603] [arXiv:1811.01324] [InSPIRE].

[28] A. Esposito, A. Pilloni and A.D. Polosa, Hybridized tetraquarks, Phys. Lett. B 758 (2016) 292 [arXiv: 1603.07667] [InSPIRE].

[29] A.M. Badalian, L.P. Kok, M.I. Polikarpov and Y.A. Simonov, Resonances in coupled channels in nuclear and particle physics, Phys. Rept. 82 (1982) 31 [INSPIRE].

[30] E. Eichten, K. Gottfried, T. Kinoshita, K.D. Lane and T.-M. Yan, Charmonium: the model, Phys. Rev. D 17 (1978) 3090 [Erratum ibid. 21 (1980) 313] [INSPIRE].

[31] E. Eichten, K. Gottfried, T. Kinoshita, K.D. Lane and T.-M. Yan, Charmonium: comparison with experiment, Phys. Rev. D 21 (1980) 203 [inSPIRE].

[32] E.J. Eichten, K. Lane and C. Quigg, Charmonium levels near threshold and the narrow state $X(3872) \rightarrow \pi^{+} \pi^{-} J / \psi$, Phys. Rev. D 69 (2004) 094019 [hep-ph/0401210] [InSPIRE].

[33] Y.S. Kalashnikova, Coupled-channel model for charmonium levels and an option for X(3872), Phys. Rev. D 72 (2005) 034010 [hep-ph/0506270] [INSPIRE].

[34] I.V. Danilkin and Y.A. Simonov, Channel coupling in heavy quarkonia: energy levels, mixing, widths and new states, Phys. Rev. D 81 (2010) 074027 [arXiv:0907.1088] [InSPIRE].

[35] I.V. Danilkin and Y.A. Simonov, Dynamical origin and the pole structure of X(3872), Phys. Rev. Lett. 105 (2010) 102002 [arXiv:1006.0211] [InSPIRE].

[36] I.V. Danilkin, V.D. Orlovsky and Y.A. Simonov, Hadron interaction with heavy quarkonia, Phys. Rev. D 85 (2012) 034012 [arXiv:1106.1552] [InSPIRE].

[37] A.M. Badalian and B.L.G. Bakker, Dynamics of the quark-antiquark interaction and the universality of Regge trajectories, Phys. Rev. D 100 (2019) 034010 [arXiv:1901.10280] [INSPIRE].

[38] A.M. Badalian and B.L.G. Bakker, Radial and orbital Regge trajectories in heavy quarkonia, Phys. Rev. D 100 (2019) 054036 [arXiv: 1902.09174] [InSPIRE].

[39] A.Y. Dubin, A.B. Kaidalov and Y.A. Simonov, The QCD string with quarks. 1. Spinless quarks, Phys. Atom. Nucl. 56 (1993) 1745 [Yad. Fiz. 56 (1993) 213] [hep-ph/9311344] [INSPIRE].

[40] A.Y. Dubin, A.B. Kaidalov and Y.A. Simonov, Dynamical regimes of the QCD string with quarks, Phys. Lett. B 323 (1994) 41 [inSPIRE].

[41] Y.A. Simonov, Exact relativistic Green's functions for the time-independent potentials, Phys. Rev. D 99 (2019) 096025 [arXiv: 1902.05364] [INSPIRE].

[42] S.S. Afonin, Towards understanding spectral degeneracies in nonstrange hadrons. Part I. Mesons as hadron strings versus phenomenology, Mod. Phys. Lett. A 22 (2007) 1359 [hep-ph/0701089] [INSPIRE].

[43] S.S. Afonin and T.D. Solomko, The large- $N_{c}$ limit of borelized spectral sum rules and the slope of radial Regge trajectories, Int. J. Mod. Phys. A 33 (2018) 1850115 [arXiv: 1805.03089] [INSPIRE].

[44] Y.A. Simonov, Relativistic path integral and relativistic Hamiltonians in QCD and QED, Phys. Rev. D 88 (2013) 025028 [arXiv:1303.4952] [InSPIRE]. 
[45] Y.A. Simonov, Spin interactions in mesons in strong magnetic field, Phys. Rev. D 88 (2013) 053004 [arXiv: 1304.0365] [INSPIRE].

[46] Y.A. Simonov, Exact relativistic Green's functions for the time-independent potentials, Phys. Rev. D 99 (2019) 096025 [arXiv: 1902.05364] [INSPIRE].

[47] M.S. Lukashov and Y.A. Simonov, Scalar mesons in a chiral theory with quark degrees of freedom, Phys. Rev. D 101 (2020) 094028 [arXiv: 1909.10384] [INSPIRE].

[48] A.M. Badalian, M.S. Lukashov and Y.A. Simonov, Relativistic Cornell-type mechanism of exotic scalar resonances, Phys. Rev. D 102 (2020) 094012 [arXiv:2001.07113] [INSPIRE].

[49] Y.A. Simonov, Di-pion decays of heavy quarkonium in the field correlator method, Phys. Atom. Nucl. 71 (2008) 1048 [arXiv:0711.3626] [INSPIRE].

[50] Y.A. Simonov, Relativistic theory of string breaking in QCD, Phys. Rev. D 84 (2011) 065013 [arXiv:1103.4028] [INSPIRE].

[51] LHCb collaboration, Observation of $J / \psi \phi$ structures consistent with exotic states from amplitude analysis of $B^{+} \rightarrow J / \psi \phi K^{+}$decays, Phys. Rev. Lett. 118 (2017) 022003 [arXiv: 1606. 07895] [INSPIRE].

[52] CDF collaboration, Observation of the $Y(4140)$ structure in the $J / \psi \phi$ mass spectrum in $B^{ \pm} \rightarrow J / \psi \phi K^{ \pm}$decays, Mod. Phys. Lett. A 32 (2017) 1750139 [arXiv:1101.6058] [INSPIRE].

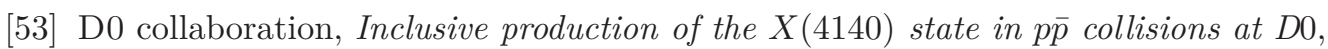
Phys. Rev. Lett. 115 (2015) 232001 [arXiv:1508.07846] [INSPIRE].

[54] CMS collaboration, Observation of a peaking structure in the $J / \psi \phi$ mass spectrum from $B^{ \pm} \rightarrow J / \psi \phi K^{ \pm}$decays, Phys. Lett. B 734 (2014) 261 [arXiv:1309.6920] [InSPIRE].

[55] BESIII collaboration, Observation of a charged charmonium-like structure in $e^{+} e^{-} \pi^{+} \pi^{-} J / \psi$ at $\sqrt{s}=4.26 \mathrm{GeV}$, Phys. Rev. Lett. 110 (2013) 252001 [arXiv:1303.5949] [INSPIRE].

[56] BeLLe collaboration, Study of $e^{+} e^{-} \rightarrow \pi^{+} \pi^{-} J / \psi$ and observation of a charged charmonium-like state at Belle, Phys. Rev. Lett. 110 (2013) 252002 [Erratum ibid. 111 (2013) 019901] [arXiv: 1304.0121] [INSPIRE].

[57] T. Xiao, S. Dobbs, A. Tomaradze and K.K. Seth, Observation of the charged hadron $Z_{c}^{ \pm}(3900)$ and evidence for the neutral $Z_{c}^{0}(3900)$ in $e^{+} e^{-} \rightarrow \pi \pi J / \psi$ at $\sqrt{s}=4170 \mathrm{MeV}$, Phys. Lett. B $\mathbf{7 2 7}$ (2013) 366 [arXiv:1304.3036] [INSPIRE].

[58] BESIII collaboration, Observation of $e^{+} e^{-} \rightarrow \pi^{0} \pi^{0} h_{c}$ and a neutral charmonium-like structure $Z_{c}(4020)^{0}$, Phys. Rev. Lett. 113 (2014) 212002 [arXiv:1409.6577] [INSPIRE].

[59] BESIII collaboration, Observation of a charged charmonium-like structure $Z_{c}(4020)$ and search for the $Z_{c}(3900)$ in $e^{+} e^{-} \rightarrow \pi^{+} \pi^{-} h_{c}$, Phys. Rev. Lett. 111 (2013) 242001 [arXiv:1309.1896] [INSPIRE].

[60] G.C. Rossi and G. Veneziano, Tetra-quarks, penta-quarks and the like: old and new views, in The hadronic vacuum polarization from lattice QCD at high precision: a topical workshop of the muon $g-2$ theory initiative, (2020) [arXiv:2011.09774] [INSPIRE].

[61] M. Anselmino, E. Predazzi, S. Ekelin, S. Fredriksson and D.B. Lichtenberg, Diquarks, Rev. Mod. Phys. 65 (1993) 1199 [INSPIRE].

[62] S. Fleck, B. Silvestre-Brac and J.M. Richard, Search for diquark clustering in baryons, Phys. Rev. D 38 (1988) 1519 [INSPIRE]. 
[63] E. Santopinto and J. Ferretti, Strange and nonstrange baryon spectra in the relativistic interacting quark-diquark model with a Gürsey and Radicati-inspired exchange interaction, Phys. Rev. C 92 (2015) 025202 [arXiv:1412.7571] [InSPIRE].

[64] C. Gutierrez and M. De Sanctis, A study of a relativistic quark-diquark model for the nucleon, Eur. Phys. J. A 50 (2014) 169 [INSPIRE].

[65] C. Alexandrou, P. de Forcrand and B. Lucini, Evidence for diquarks in lattice QCD, Phys. Rev. Lett. 97 (2006) 222002 [hep-lat/0609004] [INSPIRE].

[66] E. Megías, E. Ruiz Arriola and L.L. Salcedo, Quark-diquark string tension, excited baryonic resonances and thermal fluctuations, Phys. Scripta 95 (2020) 074005 [arXiv:2002.10871] [INSPIRE].

[67] LHCb collaboration, Observation of $J / \psi p$ resonances consistent with pentaquark states in $\Lambda_{b}^{0} \rightarrow J / \psi K^{-} p$ decays, Phys. Rev. Lett. 115 (2015) 072001 [arXiv:1507.03414] [inSPIRE].

[68] LHCb collaboration, Observation of a narrow pentaquark state, $P_{c}(4312)^{+}$, and of two-peak structure of the $P_{c}(4450)^{+}$, Phys. Rev. Lett. 122 (2019) 222001 [arXiv:1904.03947] [INSPIRE].

[69] U. Skerbis and S. Prelovsek, Nucleon-J/ $\psi$ and nucleon- $\eta_{c}$ scattering in $P_{c}$ pentaquark channels from LQCD, Phys. Rev. D 99 (2019) 094505 [arXiv: 1811.02285] [InSPIRE].

[70] H.-X. Chen, W. Chen and S.-L. Zhu, Possible interpretations of the $P_{c}(4312), P_{c}(4440)$, and $P_{c}(4457)$, Phys. Rev. D 100 (2019) 051501 [arXiv: 1903.11001] [InSPIRE].

[71] M.-Z. Liu et al., Emergence of a complete heavy-quark spin symmetry multiplet: seven molecular pentaquarks in light of the latest LHCb analysis, Phys. Rev. Lett. 122 (2019) 242001 [arXiv:1903.11560] [INSPIRE].

[72] F.-K. Guo, H.-J. Jing, U.-G. Meißner and S. Sakai, Isospin breaking decays as a diagnosis of the hadronic molecular structure of the $P_{c}(4457)$, Phys. Rev. D 99 (2019) 091501 [arXiv: 1903.11503] [INSPIRE].

[73] J. He, Study of $P_{c}(4457), P_{c}(4440)$, and $P_{c}(4312)$ in a quasipotential Bethe-Salpeter equation approach, Eur. Phys. J. C 79 (2019) 393 [arXiv:1903.11872] [InSPIRE].

[74] Z.-H. Guo and J.A. Oller, Anatomy of the newly observed hidden-charm pentaquark states: $P_{c}(4312), P_{c}(4440)$ and $P_{c}(4457)$, Phys. Lett. B 793 (2019) 144 [arXiv: 1904.00851] [INSPIRE].

[75] JPAC collaboration, Interpretation of the $\mathrm{LHCb} \mathrm{P}_{c}(4312)^{+}$signal, Phys. Rev. Lett. 123 (2019) 092001 [arXiv:1904.10021] [inSPIRE].

[76] Z.-G. Wang, Analysis of the $P_{c}(4312), P_{c}(4440), P_{c}(4457)$ and related hidden-charm pentaquark states with QCD sum rules, Int. J. Mod. Phys. A 35 (2020) 2050003 [arXiv: 1905.02892] [INSPIRE].

[77] F.-L. Wang, R. Chen, Z.-W. Liu and X. Liu, Probing new types of $P_{c}$ states inspired by the interaction between $S$-wave charmed baryon and anti-charmed meson in a $\bar{T}$ doublet, Phys. Rev. C 101 (2020) 025201 [arXiv: 1905.03636] [INSPIRE].

[78] J.-B. Cheng and Y.-R. Liu, $P_{c}(4457)^{+}, P_{c}(4440)^{+}$, and $P_{c}(4312)^{+}$: molecules or compact pentaquarks?, Phys. Rev. D 100 (2019) 054002 [arXiv: 1905.08605] [INSPIRE].

[79] J.-J. Wu, T.S.H. Lee and B.-S. Zou, Nucleon resonances with hidden charm in $\gamma p$ reactions, Phys. Rev. C 100 (2019) 035206 [arXiv:1906.05375] [INSPIRE]. 
[80] C.W. Xiao, J. Nieves and E. Oset, Heavy quark spin symmetric molecular states from $\bar{D}^{(*)} \Sigma_{c}^{(*)}$ and other coupled channels in the light of the recent LHCb pentaquarks, Phys. Rev. D 100 (2019) 014021 [arXiv: 1904.01296] [INSPIRE].

[81] M.B. Voloshin, Some decay properties of hidden-charm pentaquarks as baryon-meson molecules, Phys. Rev. D 100 (2019) 034020 [arXiv:1907.01476] [InSPIRE].

[82] Y.-J. Xu, C.-Y. Cui, Y.-L. Liu and M.-Q. Huang, Partial decay widths of $P_{c}(4312)$ as a $\bar{D} \Sigma_{c}$ molecular state, Phys. Rev. D 102 (2020) 034028 [arXiv: 1907.05097] [InSPIRE].

[83] M.-Z. Liu, T.-W. Wu, M. Sánchez Sánchez, M.P. Valderrama, L.-S. Geng and J.-J. Xie, Spin-parities of the $P_{c}(4440)$ and $P_{c}(4457)$ in the one-boson-exchange model, Phys. Rev. D 103 (2021) 054004 [arXiv: 1907.06093] [INSPIRE].

[84] M.-L. Du et al., Interpretation of the $\mathrm{LHCb} P_{c}$ states as hadronic molecules and hints of a narrow $P_{c}(4380)$, Phys. Rev. Lett. 124 (2020) 072001 [arXiv: 1910.11846] [InSPIRE].

[85] B.-T. Zhang, J.-S. Wang and Y.-L. Ma, Pentaquark states from bound state approach with chiral partner structure, arXiv:2002.10954 [INSPIRE].

[86] S.-Q. Kuang, L.-Y. Dai, X.-W. Kang and D.-L. Yao, Pole analysis on the hadron spectroscopy of $\Lambda_{b} \rightarrow J / \Psi p K^{-}$, Eur. Phys. J. C 80 (2020) 433 [arXiv:2002.11959] [inSPIRE].

[87] BESIII collaboration, Observation of a near-threshold structure in the $K^{+}$recoil-mass spectra in $e^{+} e^{-} \rightarrow K^{+}\left(D_{s}^{-} D^{* 0}+D_{s}^{*-} D^{0}\right)$, Phys. Rev. Lett. 126 (2021) 102001 [arXiv: 2011.07855] [INSPIRE]. 